Revue italienne d'études françaises

Littérature, langue, culture

$8 \mid 2018$

L'Écrivain critique de lui-même

\title{
« Faire le procès à son livre ». Épistolarité et discours d'auteur chez Étienne Pasquier
}

\section{Magda Campanini}

\section{Q OpenEdition}

\section{Journals}

Édition électronique

URL : http://journals.openedition.org/rief/2342

DOI : 10.4000/rief.2342

ISSN : 2240-7456

Éditeur

Seminario di filologia francese

Référence électronique

Magda Campanini, « «Faire le procès à son livre ». Épistolarité et discours d'auteur chez Étienne Pasquier », Revue italienne d'études françaises [En ligne], 8 | 2018, mis en ligne le 15 novembre 2018, consulté le 20 avril 2019. URL : http://journals.openedition.org/rief/2342 ; DOI : 10.4000/rief.2342

Ce document a été généré automatiquement le 20 avril 2019.

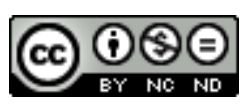

Les contenus de la RIEF sont mis à disposition selon les termes de la Licence Creative Commons Attribution - Pas d'Utilisation Commerciale - Pas de Modification 4.0 International. 


\title{
« Faire le procès à son livre ». Épistolarité et discours d'auteur chez Étienne Pasquier
}

\author{
Magda Campanini
}

1 Pourquoi choisir Étienne Pasquier dans le cadre d'une réflexion sur l'écrivain critique de lui-même ? Un peu éclipsé face à ses illustres contemporains, il a parfois été rangé, à tort, aux marges du système littéraire. Dans sa longue carrière d'homme de lettres, de juriste, d'historien, de poète et d'épistolier, il traverse les décennies allant des années 1550 à 1615, date de sa mort, fréquente avec assiduité le cercle de la Pléiade et se fait l'interprète d'une historiographie nationale en parallèle avec une historiographie littéraire dont l'enjeu principal est constitué par la valorisation du français comme langue nationale. Si Pasquier est le premier écrivain à tenter de mettre en perspective le "progrès » des lettres françaises, en jetant ainsi les fondements d'une histoire littéraire nationale, il nous offre aussi une écriture qui s'ouvre constamment sur un discours autoréflexif où se façonnent sa figure d'auteur et la conscience de son œuvre et de sa diffusion. Il suffit d'évoquer ses liens avec un vaste réseau d'imprimeurs et de libraires, notamment Abel l'Angelier à qui le lie une amitié dont nous avons témoignage jusqu'aux années 1590 et chez qui il publie ses écrits ${ }^{1}$ parfois sous pseudonyme ou anonymement ${ }^{2}$, tout en en réorganisant à plusieurs reprises la disposition pour mieux les valoriser. Pasquier a toujours mis son statut d'auteur au centre de son œuvre et il en a fait l'objet manifeste d'une construction identitaire, d'une stratégie éditoriale et d'une réflexion critique. C'est sur cette émergence de la parole auctoriale en tant que clé d'accès à la couche autoréflexive de l'écriture de Pasquier et aux mécanismes d'autopromotion de son œuvre que nous nous proposons de nous arrêter.

2 Le terrain privilégié de cette étude sera son œuvre d'épistolier, développée tout au long de sa vie et regroupée en vingt-deux livres de trois cents lettres environ, ce qui en fait « la collection la plus importante de lettres françaises en prose du XVI siècle $»^{3}$. Cependant nous limiterons notre lecture à un corpus restreint, formé de quelques missives et lettres liminaires d'ouvrages publiés entre 1555 et 1610. 
Si Pasquier a introduit dans la France de son époque ce qu'Hugo Friedrich définit comme "la lettre artistique en langue vulgaire $»^{4}$, il a pareillement fait de cette forme d'écriture l'un des lieux favorisés de la prise de parole de la part de l'écrivain. Celle-ci s'étend des appareils liminaires - lieux topiques de la mise en place des stratégies d'auteur - au corps des lettres, constituant un véritable laboratoire où se mettent en œuvre des processus d'énonciation, d'argumentation et d'emprise sur le lecteur relevant de la construction de l'ethos auctorial. C'est pourquoi nous nous proposerons de saisir le reflet de l'œuvre au sein de la parole d'auteur, de mettre en relation cette émergence avec les spécificités de l'énonciation épistolaire et d'évaluer l'influence du discours d'auteur sur la réception de l'œuvre.

4 La question de la reconnaissance et de l'appropriation de l'identité auctoriale se lie à la relation dialectique entre image publique et image privée du sujet écrivant et se précise ultérieurement suivant la typologie textuelle adoptée. Dans le cas de Pasquier, cet aspect est d'autant plus visible qu'il se lie à un écrivain dont le poids politique et l'activité publique - n'oublions pas qu'il était avocat général du roi à la Chambre des comptes s'associent à un réseau de proches très étendu, ce qui offre une matière riche et fonctionnelle à l'autoréflexion dans une écriture épistolière familière pratiquée dans le sillage des grands maîtres de l'Antiquité tels que Cicéron ou Pline, mais aussi de Pétrarque, Politien, Érasme, Budé. Il s'agit d'une épistolarité qualifiée de « familière » non dans le sens des relations parentales ou amicales avec les destinataires, mais parce que, d'un point de vue stylistique, elle se distingue par des tournures assez souples et par le refus d'un formalisme excessif ${ }^{5}$. Certes, les lettres de Pasquier s'ouvrent sur un vaste éventail de sujets touchant à l'histoire, à la politique et au droit, mais sa production compte également des lettres d'amitié, parfois au ton ludique, des lettres-chronique, des lettres de circonstance et des lettres d'amour. Pasquier lui-même met l'accent sur la variété des «produits» épistolaires qu'il offre au lecteur et qu'il définit comme «une denrée meslée ", composée de "toute sorte de marchandises ${ }^{6}$. Cette prise de parole de l'écrivain-épistolier visant à mettre en valeur un aspect essentiel de son œuvre épistolaire s'affirme avec une efficacité particulière dans la lettre à Antoine Loysel qui inaugure le recueil des Lettres d'Estienne Pasquier, dont la première édition parut en 1586, du vivant de l'auteur7. Dans ce volume dépourvu d'avis au lecteur et d'épître dédicatoire, cette lettre fait fonction de préface, se situant dans l'espace où, par tradition, l'écrivain exhibe une identité énonciative qui lui est propre et, simultanément, marque sa position dans le champ littéraire. C'est précisément ce que fait Pasquier, qui assume sa fonction d'auteurépistolier et prend parti pour le genre dans lequel il s'est investi, en revendiquant sa légitimité à partir du titre de son ouvrage: Lettres d'Estienne Pasquier. Le mot Lettres y apparaît sans la qualification qui indiquerait la fonctionnalité ou le didactisme dont elles seraient porteuses, comme c'est le cas, par exemple, pour les catégories, très à la mode, des manuels ou des compilations. D'entrée de jeu, Pasquier met en évidence la « pauvreté » de ce titre, qui - écrit-il - est censé être considéré comme « trop bas » par les lecteurs et devrait susciter leur réprobation. Cette considération fait apparaittre la figure d'un lecteur virtuel représenté comme expression du lectorat français dont l'auteur conjecture - c'est là un autre topos préfaciel - d'éventuelles critiques ou des réserves touchant à la forme d'un ouvrage dont la charge novatrice serait vue comme déplorable en ce qu'elle se fonde sur l'audace de "mettre ses missives sur la monstre». Nous assistons là à la mise en œuvre d'une stratégie d'auteur visant au renversement d'un préjugé de lecture fondé sur une doxa littéraire qui disqualifie le discours épistolaire 
d'auteur en langue vernaculaire. La démarche argumentative suit un procédé de dissociation selon lequel la raison du refus de son ouvrage par des "controlleurs" (comme Pasquier les définit à la fin de cette lettre) devient l'aspect qui valorise ce genre d'écriture et justifie sa mise en livre. En ayant recours à l'argument d'autorité, il place son œuvre dans la lignée de celles des grands auteurs - en premier lieu les Grecs et les Romains - reconnus par «toutes autres nations qui ont fait profession de bien dire ». Il arrive ainsi à une définition de cette lignée littéraire dont il se veut le promoteur et l'initiateur en France et qui consiste à « exposer au public les lettres que les gens de marque s'entrescrivoyent privément $»^{8}$.

Par le biais de l'autoréflexivité, la composition épistolaire de Pasquier s'ordonne sous nos yeux selon des axes qui en tracent les lignes de sens et en justifient la publication en tant qu'ouvrage accompli. Pasquier se définit comme un "hardy entrepreneur » qui, en publiant ses lettres, aborde un sujet «non accoustumé à la France». Il souligne que, malgré des prédécesseurs illustres (« Un Erasme et un Budé, lumières de notre siècle »), il leur est en quelque sorte supérieur en raison de son choix d'écrire « au langage auquel j'ay esté allaicté dés la mammelle de ma mere. Me promettant - poursuit-il - que si nostre langue prend pied entre les nations estranges, je leur pourray servir d'exemple non adopté »". Le défaut qu'il reproche aux épistoliers modernes ayant préféré le latin au français est d'avoir recouru à "plusieurs traits de parler mal couchez, mal limez, mal appropriez, comme de la part de ceux qui les accomodoient plus à la liberté de leur esprit, qu'à la pureté du langage, ores que le principal but de ceux qui escrivent en ce genre doive estre l'embellissement de la langue en laquelle ils descouvrent leurs sens ${ }^{10}$. La promotion d'une épistolarité en vulgaire passe par l'attribution d'un statut de littérarité à une forme d'écriture conçue par les auteurs classiques dans leur propre langue et qui se propose comme modèle inédit à suivre maintenant en langue française.

6 Le terrain qui nourrit l'autocommentaire d'auteur s'élargit aux faits stylistiques. Sous le guide de l'écrivain-épistolier, la diversité des lettres, voire leur hétérogénéité, révèle des traits unifiants. Si les lettres sont jugées comme des «minutes, esparses ça et là, comme d'un naufrage $»^{11}$, conformément au topos modestiae et à la rhétorique de la justification du projet littéraire, elles présentent une souplesse de style et une légèreté d'expression dues au rejet de la surabondance oratoire qui caractérise généralement les formules conventionnelles d'ouverture et de clôture. À son destinataire d'élection et double du lecteur, l'ami Loysel, l'auteur illustre sa stratégie, qui consiste à libérer ses lettres des incrustations rhétoriques vides de sens :

J'adjousteray que mettant la main à cest oeuvre je me delibere de luy oster la teste \& les pieds : Je veux dire ces mots de Monseigneur, Monsieur, \& autres dont nous faisons les premiers frontispices de nos lettres: \& plus encore ceste closture des quatre \& cinq lignes de recommandations aux bonnes graces, qui ne servent que de perte de temps, \& remplissage de papier. [...] Chose qui ne plaira pas de prime face au peuple, comme nouvelle \& inacoustumée entre nous. ${ }^{12}$

7 Il est presque superflu de rappeler que dans ce refus des formules figées et des contraintes conventionnelles du protocole épistolaire nous retrouvons l'écho de Montaigne qui observe que «les lettres de ce temps sont plus en bordures et prefaces, qu'en matiere » et qui, en évoquant sa propre manière, ne cache pas son intolérance pour toute forme de cérémonial stérile :

Comme j'ayme mieux composer deux lettres que d'en clorre et plier une, et resigne tousjours cette commission à quelque autre: de mesme, quand la matiere est achevée, je donrois volontiers à quelqu'un la charge d'y adjouster ces longues 
harengues, offres et prieres que nous logeons sur la fin, et desire que quelque nouvel usage nous en descharge $;[. ..] .^{13}$ en quelque sorte à Montaigne le «nouvel usage» que ce dernier souhaite tout en reconnaissant dans sa démarche une tentative "nouvelle \& inacoustumée entre nous". Sa conscience stylistique va de pair avec la conscience de la valeur novatrice de son projet: il juge son œuvre d'épistolier comme un tournant dans l'histoire de l'épistolographie aussi bien sur le plan générique - la lettre familière vernaculaire de dérivation érasmienne - que sur celui du style, préférant la sincérité de l'expression au "masque d'oraison », c'est-à-dire à tout excès d'artifice oratoire. Certes, Pasquier se présente comme l'initiateur d'un nouveau genre, mais à l'exception des Dames de Roche, il passe sous silence les pratiques épistolaires qui ont devancé la sienne ou qui lui sont contemporaines. S'il ne fait pas mention d'Hélisenne de Crenne, ni de Gabriel Chappuys, ni même des Lettres missives et familières d'Étienne Du Tronchet, sans doute parce que ces dernières s'inscrivent dans le cadre des manuels épistolaires, ou que, dans le cas d'Hélisenne de Crenne, elles participent d'un échange demi-fictif, c'est surtout parce qu'il vise à se distinguer par la conscience et le souci d'« illustrer » et d'enrichir le français et de se poser en tant que modèle d'auteur épistolaire écrivant en langue vulgaire, au point que la critique a pu évoquer son « nationalisme littéraire ${ }^{15}$.

commentaire de l'auteur sur son livre offre aux lecteurs des considérations sur les sujets des lettres et sur leur agencement. Après en avoir écarté les sujets domestiques et strictement privés - ce qui donne à son œuvre épistolaire un statut littéraire - il suggère d'associer la variété des tons et des sujets de ses lettres aux différentes étapes de sa vie qui ont porté à leur rédaction. Une sorte de narrativité allusive affleure parmi les pages de son livre qui serait lisible comme

un tableau general de tous mes aages, dans lequel vous verrez icy mon Printemps, là mon Esté, puis mon Automne tiré au vif, je veux dire mes lettres moulées sur le patron des aages qui ont diversement commandé à mes opinions: $\mathrm{Ne}$ m'estant proposé maintenant de contenter seulement les sages, mais aussi les fols. ${ }^{16}$

La sagesse et la folie évoquées à la dernière ligne sont un corollaire thématique important, une polarité double autour de laquelle se disposent les lettres, deux axes d'agrégation que l'auteur introduit pour donner aux lecteurs - qu'ils soient sages ou fous - le moyen de se reconnaître dans ses écrits et, en même temps, de porter un jugement indulgent sur des lettres qui n'adoptent pas la gravité qui convient au sujet ou au ton. Cette évocation de la «folie » est fortement présente dans les lettres où Pasquier commente et juge son œuvre et invite le lecteur à le suivre dans son parcours. Elle est associée tantôt à la jeunesse, tantôt à la vieillesse et se lie à l'expérience de l'amour. J'en considérerai les occurrences dans un ouvrage épistolaire de jeunesse et dans le recueil de 1586.

11 Dans le premier cas, vingt-quatre lettres à sujet amoureux paraissent en 1555 dans le Recueil de rymes et proses de E. P. Regroupées dans une section au titre anodin, Epistres, elles constituent en réalité une narration par lettres où, sous le nom d'Étienne Pasquier, un narrateur-épistolier qui se trouve à la merci d'une femme cruelle et trompeuse évoque ses souffrances, poursuivant une réflexion sur l'amour que l'auteur avait inaugurée dans le Monophile $e^{17}$.

Dès son premier coup d'essai épistolaire, Pasquier met en évidence l'unicité de son entreprise, qui consiste à publier des lettres témoignant d'une sorte de descente aux 
enfers de la passion, qualifiée par lui de «folie ». L'accès offert au public à la sphère personnelle et à l'expérience de la passion par le biais d'un discours - le discours par lettres - qui, par ses enjeux communicatifs, est censé être ancré dans une expérience réelle, est l'élément novateur que le narrateur met en valeur dans la première de ces epistres $^{18}$. La lettre d'ouverture - à fonction préfacielle - est l'espace où l'auteur se penche sur l'ouvrage qu'il confie à ses lecteurs et sur lequel il porte un regard critique visant à attirer la bienveillance du public et à lui offrir des clés de lecture. Dans cet ouvrage de jeunesse, l'auteur cherche déjà à promouvoir sa figure et son parti pris d'innovation, qui consiste ici à offrir ses amours - dont il s'est repenti - en spectacle à ses lecteurs : "Qui eust jamais estimé que telle eust esté la sottie d'un homme, de non seulement estre fol, \& avoir cognoissance de sa folie, mais aussi d'appeter que le monde en eust cognoissance ?» 19 .

Une double ambiguïté s'instaure dans cette lettre-préface: l'une, rhétorique, entre discours autojustificatif et discours autocélébratif (ce qui rentre à plein titre dans le topos préfaciel) et l'autre portant sur le statut des lettres : s'agit-il de lettres authentiques ou de lettres fictives?

C'est une histoire, m'en croyez, une histoire de ma folie \& ne dressay oncques ces lettres qu'ainsi ou qu'amour, ou que desdain les dictoit: desquelles aucunes furent (peut-estre) envoyées, les autres non, \& les unes \& les autres, seulement faites pour plaisir, furent basties soubs la charge de ces deux trahistres capitaines, qui à l'envy ont commandé sur mes esprits. [...]. ${ }^{20}$

14 La folie d'amour est-elle la trace d'un vécu ou le fruit d'une invention littéraire ? Et cette folie, en quoi consiste-t-elle? En un aveuglement induit par la passion ou en la présomption arrogante d'un auteur qui a la prétention d'inventer sciemment une forme nouvelle, la fiction amoureuse par lettres? Laissons la question ouverte, non sans souligner que, quoi qu'il en soit, dans cet espace autoréflexif qui évoque une « histoire ", l'auteur s'investit du rôle de narrateur et le fait devant ses lecteurs ${ }^{21}$. Pasquier assure aussi dans son œuvre une continuité qui la traversera au fil des ans, en vertu de laquelle il ne cessera de juger le discours épistolaire come cadre possible d'une narration ou d'une insertion narrative, s'autorisant la publication d'échanges épistolaires fictifs ${ }^{22}$. Ainsi, dans le recueil de 1586, il invite à composer des lettres « [en les ] envoyant [...] ou [en] feignant de les envoyer aux uns et aux autres, sans exclure « quelque honorable narré »; l'objectif en sera le plaisir et l'instruction du lecteur («profit \& plaisir ensemble ») ${ }^{23}$.

L'assomption du rôle d'auteur lui permet également d'évaluer l'accueil de son œuvre de la part $\mathrm{du}$ lectorat et d'orienter sa réception. Deux lettres sont particulièrement importantes à cet égard. Publiées en 1586 et adressées à Louis Buisson, elles contiennent une analyse articulée et un jugement $a$ posteriori sur les Epistres de 1555. Trente ans après, leur auteur ne les désavoue $\operatorname{pas}^{24}$; bien au contraire, il renforce son avis positif et il reconstruit, non sans complaisance, leur fortune éditoriale qui sépare leur publication du présent de l'écriture :

Lors que je les [les Lettres amoureuses] fis imprimer, je ne mis mon nom sur le frontispice du livre, pour sonder, avecques moins de hazard de ma reputation, quel seroit le jugement du peuple. Et de fait j'ay long temps depuis estimé que la memoire en fust perdue, toutes-fois puis n'agueres feuilletant quelques livres en la boutique de L'Angelier, je trouvay qu'on les avoit fait rimprimer avec celles de Parabosco Italien, et qui plus est que l'on avoit mis contre ma volonté, mon nom. Qui me fit penser qu'elles avoyent eu meilleur succès que ne m'estois promis. ${ }^{25}$ 
Eistres - désormais restituées à la paternité de leur auteur - ont en effet été éditées pour la deuxième fois, sans date, à l'intérieur des Lettres amoureuses de Parabosque traduites en français ${ }^{26}$. Tout au long de son œuvre, nous l'avons vu, Pasquier a eu recours à plusieurs reprises à l'anonymat et au pseudonyme ${ }^{27}$ (c'est le cas de La Puce, de La main, des Ordonnances d'Amour, du Catéchisme des jésuites et de La jeunesse), sans oublier pourtant de rétablir a posteriori sa paternité ni d'élaborer des rééditions de ses ouvrages à travers le réagencement des pièces qui les composent. L'attention permanente qu'il porte à son œuvre et à son polissage se complète par le soin qu'il a d'en retracer le parcours dès la sortie de l'atelier de l'imprimeur. La vision quasi-physiologique d'une création littéraire qui, à partir de l'image du livre-enfant, suit et reproduit le parcours des âges de la vie s'ébauche dans la lettre-préface des Epistres de 1555 et s'élargit dans les deux lettres à Buisson. L'écrivain-épistolier invite à voir dans la résonnance émotive de cette histoire par lettres la marque de la jeunesse dont elle est l'expression et emphatise la valeur novatrice de ce « livre » qui a enrichi les formes de l'écriture littéraire en prose :

vrayement, vous avez raison de m'improperer maintenant qu'en ma jeunesse, à la suite de mon Monophile, j'aye mis en lumiere, un livre d'Epistres amoureuses : ce qui n'avoit encores esté attenté par nul des nostres; comme si vous ne sçaviez pas bien que tout ainsi que chaque saison de l'année, aussi faut-il que chaque aage ait ses fonctions particulières.

[...] Je vous accorde que ces lettres estoient une vraye folie. Mais [...] c'estoit une belle folie dont oiseux, je trompois l'oisiveté de ma jeunesse par faulte de meilleur subject. $^{28}$

L'image de la jeunesse portée à la passion et à l'écriture amoureuse entre en résonance avec celle de la vieillesse. Dans la continuité de ses autocommentaires de jeunesse, l'acte critique réflexif de Pasquier justifie et valorise l'écriture à sujet amoureux même dans ses formes les moins reconnues et les moins « hautes » telles que la narration d'amour par lettres ou, filant la métaphore d'une œuvre construite comme un théâtre des âges de la vie, dans sa Pastorale du vieillard amoureux. Dans la lettre-préface adressée à M. Airault, Lieutenant criminel au Siège présidial d'Angers, Pasquier raconte la genèse de cet ouvrage de vieillesse et, ébauchant une scène de tribunal, il se défend des imputations en revendiquant son droit à pratiquer une littérature de récréation. La « folie » qu'il évoque encore une fois est ici une folie de vieillesse, un amour sénile métaphore d'une écriture enjouée que la plume de l'auteur justifie à priori devant ses lecteurs et pour la postérité :

Si vous voulez estre Lieutenant criminel implacable, ne m'imputez à faute, si avecques tout mon bien j'aye aussi perdu mon esprit. Mais à quel propos toutes ces excuses? Je veux à face descouverte qu'on sçache que je fay le fol. [...] Voyez doncques ce veillard entrer maintenant sur le theatre pour jouer son personnage, et monstrer, tant en prose qu'en vers, qu'il n'est gueres sage. ${ }^{29}$

Il peut sembler paradoxal que cette pastorale du vieillard ait été publiée dans un recueil intitulé La jeunesse d'Estienne Pasquier. L'écriture d'évasion et à sujet amoureux est pourtant - on l'a vu - le lien entre la folie de jeunesse et la folie de vieillesse, un lien d'autant plus fort qu'il présuppose que ce type d'écriture soit lisible comme une représentation des passions et des émotions. Ce recueil, paru vers la fin de la vie de l'auteur, est un exemple intéressant d'une stratégie d'auteur fondée sur le choix d'une catégorie éditoriale à succès, le florilège, ici présenté sous la métaphore conventionnelle du "bouquet», dans le but de proposer aux lecteurs une sélection d'œuvres et d'en dresser une sorte de bilan. Le principe qui gouverne cette sélection assure la cohérence interne de l'ensemble : les ouvrages réunis dans La jeunesse permettent de suivre la vie de l'auteur et d'en rapporter une image non officielle, celle d'un auteur qui, par intervalles, 
laisse de côté la gravité de ses occupations pour s'adonner à l'écriture amoureuse et d'invention, et se consacrer à la composition «de quelques vers par maniere de passetemps $»^{30}$. En dépit du titre, cette compilation suit toutes les saisons de la vie et de la production de l'auteur, du Monophile aux Lettres amoureuses, des jeux poétiques à la Pastorale du vieillard amoureux. Le signataire de l'avis au lecteur, André Du Chesne Tourangeau, illustre le critère qui a gouverné la mise en livre de La jeunesse :

[...] ce sont les fleurs de sa primevère dont j'ay voulu faire ce bouquet que j'ay aussi enjolivé de quelques fruits de son Esté, et Automne [...] Je me promets que si les jeunes fleurs esparses çà et là, t'ont esté autresfois agreables, estant maintenant reduites en un corps, elles te seront d'une plus souefve odeur. Sous ceste esperance, ay-je voulu donner à ce mien Recueil le nom de La Jeunesse d'Estienne Pasquier, ores qu'outre les premieres gayetez de cest aage, j'ay compris plusieurs autres pieces qui depuis se sont escoulées de sa plume. Et croy que je n'en seray [a2 $\left.\mathrm{v}^{\circ}\right]$ par luy desavoué. Ayant en cecy pour parrein le grand \& docte Cardinal Bembo, honneur de son siecle, qui ne doubta d'adjouster à ses œuvres de poix toutes les belles faillies \& gaillardises de son esprit sous le titre de Petri Bembi Iuvenilia. ${ }^{31}$

Précisons que derrière le nom d'André Du Chesne se cache Pasquier, comme il le révélera lui-même deux ans plus tard, dans la lettre-testament à M. de Sainte-Marthe qui accompagne l'envoi « à face ouverte » de ce même livre ${ }^{32}$. S'il se déguise en éditeur, c'est qu'il souhaite guetter de loin, prudemment, les réactions des lecteurs au moment où, par le biais d'une personne tierce, il leur transmet ${ }^{33}$ un «bilan » de son œuvre et dirige leur intérêt vers ses compositions "mineures », en les présentant comme l'expression des différents moments de la vie de l'auteur et comme un divertissement d'homme de lettres. La conscience de sa valeur d'écrivain dont la plume n'a jamais été « oiseuse » se confirme et trouve ses lettres de noblesse dans le rapprochement de ce recueil avec un modèle reconnu du genre, les Iuvenilia de Pietro Bembo. La variété de sujets et de styles qui fait la spécificité de l'écriture de Pasquier n'est pas sans rapport avec le rappel constant d'une création littéraire qui représente sur le papier la vie d'un écrivain à la plume « diversifiée selon la diversité de ses ans ». Ce dernier aspect, remarqué dans les liminaires des Lettres amoureuses, émerge encore dans un autre péritexte important, l'avis au lecteur des Jeux poétiques, toujours publiés dans La Jeunesse. L'auteur y invite le lecteur virtuel à suivre les détours de son œuvre : un parcours tortueux qui le désorientera, mais qui offre à l'auteur l'occasion de poser la question du sens de son œuvre (« Mais, en bonne foy, que dois-tu juger de ceste meslange ? $\left.\aleph^{34}\right)$. Pasquier ne laisse pas son lecteur sans réponse et lui offre sa propre interprétation : «Si tu es homme d'entendement, tu diras que c'est un theatre des affections humaines que j'ai voulu représenter sous ma personne $»^{35}$. L'allusion au topos du théâtre du monde suggère une lecture qui présente l'expérience subjective de l'auteur en ce qu'elle a d'universel, sans cacher pour autant une posture d'autoappréciation $^{36}$.

Se pencher sur la prise de parole auctoriale chez Pasquier revient à mettre en valeur la fonction de la lettre en tant que lieu et moyen privilégié de l'autocommentaire. Le dialogisme étant une des structurations majeures du discours par lettres, le dialogue écrivain-lecteur ne fait que dédoubler le rapport d'interlocution entre l'épistolier et son destinataire au premier degré par l'incorporation d'une instance virtuelle de réception (celle du lecteur à venir), à la laquelle l'épistolier confie ses observations sur ses propres ouvrages et à qui il lègue son identité d'auteur. La dynamique épistolaire démultiplie forcément les lieux de l'émergence de la parole auctoriale et en tisse un réseau ramifié d'occurrences qui souvent - on l'a vu - se répondent entre elles, non sans épaissir 
l'opacité du discours d'auteur. La production épistolaire de Pasquier accompagne d'ailleurs constamment sa longue activité d'homme de lettres et, pour cette raison aussi, elle est un lieu d'enquête privilégié et fécond pour la critique. Nous avons vu que ce qui émerge avec évidence au fil des prises de parole de l'écrivain-épistolier est surtout le caractère novateur de son «entreprise » de publication, aussi bien pour ce qui est des formes de discours littéraire qu'il véhicule et qu'il légitime - notamment la lettre familière et la fiction par lettres - que pour son engagement dans la promotion d'une épistolarité littéraire en langue française. Pasquier adopte une prise de parole auctoriale autoritaire, assumée sur un ton péremptoire visant à faire exister son œuvre selon sa conception et à assurer un contrôle sur la postérité du texte. En témoignent la centralité du projet d'autocélébration et de promotion des traits d'originalité de son œuvre par laquelle il veut se faire reconnaître comme le plus novateur de «tous ces modernes qui redigerent leurs fantaisies ${ }^{37}$ en latin. En témoigne aussi la planification des éditions de ses lettres - une dizaine en France et à l'étranger entre 1586 et l'édition posthume de 1619 - auxquelles s'ajoute la grande édition des CEuvres de 1723. Les mécanismes d'autoappréciation dépassent les frontières de sa production épistolaire et s'étendent à une partie bien plus large de sa production, comme le montre la lettre à Saincte-Marthe déjà citée, où il envisage un programme détaillé de publications et de réimpressions tant de son vivant qu'après sa mort. Pasquier tient particulièrement au sort éditorial de ses lettres, qui «ont pris leur vol non seulement par la France, ains en plusieurs nations estranges ${ }^{38}$ et dont il annonce dix livres sous presse.

Si de son vivant et dans les décennies suivantes il a joui d'une grande réputation et a conditionné le marché libraire, dans les années 1660 La Bibliothèque françoise de Sorel porte un jugement hâtif et dépréciatif sur le style de ses lettres : à l'exclusion des lettres s'apparentant à des chroniques historiques, dont l'intérêt est attribué au sujet, les autres ne sont considérées par Sorel comme ni «élégantes » ni « polies ». Furetière, de son côté, souligne «le tour guindé » de ses lettres d'amour ${ }^{39}$. Au XVIII ${ }^{e}$ siècle, si Rigoley de Juvigny loue Pasquier pour ses "talents divers ${ }^{40}$ ", Titon du Tillet dans Le Parnasse françois lui reproche de donner "trop de licence » à son caractère enjoué " comme il paroît dans

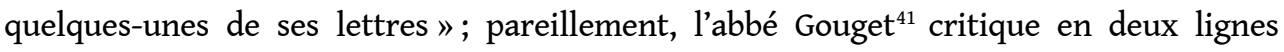
l'excès d'enjouement de certaines lettres, de même que le peu de valeur et « l'indécence " de tous ses ouvrages à sujet amoureux. On ne peut parler de doxa critique sur les Lettres de Pasquier qu'à partir du XIX siècle, lorsque paraît en 1849 l'anthologie de Léon Feugère qui s'attache à classer les lettres selon leur sujet, mais surtout à partir de Sainte-Beuve. Dans une de ses Causeries du lundi (6 janvier 1851), Sainte-Beuve analyse en détail l'œuvre de Pasquier et s'arrête assez longuement sur ses lettres, « premier recueil de ce genre qui ait paru dans notre langue ». Cette image de Pasquier initiateur absolu de la tradition épistolaire en langue française reflète celle que Pasquier lui-même exhibe dans son œuvre. Il en va de même pour la vision d'ensemble de ses lettres qui - écrit Saint-Beuve «le montrent au vrai dans la succession de ses âges [...] $»^{42}$. Cette image reproduit celle de la première lettre de l'édition L'Angelier de 1586 et rend ainsi lisible à distance l'empreinte de la réflexion d'auteur sur son commentaire critique. L'exégèse la plus récente a élargi le terrain d'enquête aux stratégies rhétoriques, aux dynamiques éditoriales et à la théorie des genres. L'œuvre épistolaire de Pasquier en ressort comme un «corps $»^{43}$ organique, quoique bigarré au plan de l'articulation, des tons et des contenus. Les réflexions métadiscursives de Pasquier ont retenu l'attention de la critique contemporaine : c'est au fil de son autocommentaire d'auteur qu'on suit la construction d'une conscience littéraire exprimant la promotion d'un modèle de prose française et 
d'une esthétique du mélange et des formes ouvertes qui, dans le champ épistolaire, se traduit par un projet clairvoyant de refondation de la forme-lettre.

\section{NOTES}

1. Les rapports de Pasquier avec les libraires parisiens et de province sont riches et complexes, d'autant plus que dans sa longue carrière d'écrivain il s'est toujours soucié du « sort » et de la survie de ses ouvrages. Pour cet aspect nous renvoyons à C. Magnien, Étienne Pasquier (1519-1615) et l'édition de ses ceuvres, Szeged, Scriptum, 1999, p.3-24. De la même année date la thèse d'habilitation de C. Magnien sur « Pasquier épistolier », non publiée.

2. Voir C. Magnien, «Réflexions sur l'anonymat au XVI ${ }^{\mathrm{e}}$ siècle : l'exemple d'Étienne Pasquier (1529-1615) », dans Littérales, 39, 2007, p. 9-28.

3. L. Vaillancourt, La lettre familière au XVI siècle. Rhétorique humaniste de l'épistolaire, Paris, Champion, 2003, p. 357.

4. H. Friedrich, Montaigne, Paris, Gallimard, 1968, p. 371.

5. Nous renvoyons en particulier à l'étude de Luc Vaillancourt déjà citée et, du même auteur, à l'article « Les Lettres d'Étienne Pasquier : du familier à l'informel », dans Dalhousie French Studies, 85, 2008, p. 27-34. Voir aussi G. Gueudet, L'Art de la lettre humaniste, Paris, Champion, 2004, p. $410-411$.

6. É. Pasquier, «Lettre I. A M. Loysel Advocat en la Cour de Parlement de Paris. », dans Id., Les CEuvres d'Estienne Pasquier, Amsterdam, Compagnie des Libraires associés, 1723, t. II, liv. I, col [onne] 4 (dorénavant Euvres). Plus loin dans cette même lettre il annonce : « Vous en trouverez les aucunes serieuses, les autres gayes, autres folastres, autres accompagnées de discours \& les autres n'avoir plus beau subjet, sinon qu'elles sont sans subjet \& comme fleches descochées à coup perdu ». Sauf indication contraire, toutes nos citations feront référence à cette édition.

7. Id., Les Lettres d'Estienne Pasquier, Paris, A. L'Angelier, 1586 ; plus d'une trentaine d'exemplaires de cette édition ont été recensés, ce qui en montre l'importance (voir C. Magnien, "Étienne Pasquier et l'édition de ses œuvres ", cit., p. 18, n. 85).

8. É. Pasquier, Euvres, cit., t. II, Lettre I, col.1.

9. Ibid., col. 3-4.

10. Ibid., col. 3.

11. Ibid., col. 2.

12. Ibidem.

13. Montaigne, Essais, I, 40. Pour la revendication d'une écriture qui refuse toute linéarité contraignante voir en particulier M. Magnien, «Un écho de la querelle cicéronienne à la fin du $\mathrm{XVI}^{\mathrm{e}}$ : éloquence et imitation dans les Essais ", dans F. Lestringant (dir.), Rhétorique de Montaigne, Paris, Champion, 1985, p. 85-99. L'écho de Montaigne se fait entendre à plusieurs reprises chez Pasquier; c'est le cas des pages sur les cannibales, retravaillées en partie dans la lettre à M. de Querquisinen sur les «Terres neufves » (dans CEuvres, cit., t. II, liv. III, Lettre 3, col. 55-56) pour laquelle nous renvoyons aux études de F. Lestringant ( « La négation des Cannibales, Montaigne et Pasquier ", dans M. Tetel et G. Mallary Masters (dir.), Le parcours des Essais, Paris, Aux amateurs de livres, 1989, p. 225-237 et Le cannibale : grandeur et décadence, Genève, Droz, 2016, p. 164), de la reprise du thème de l'intelligence animale de l'« Apologie de Raymond Sebond » dans la « Lettre 
en forme de paradoxe pour les bestes brutes » (Euvres, cit., t. II, liv. X, Lettre 1, col. 249-260), sans compter - sur le plan du discours - le réemploi de certaines métaphores.

14. Voir C. Magnien-Simonin, «Étienne Pasquier lecteur de Montaigne », dans Cl.-G. Dubois (dir.), Montaigne et Henri IV (1595-1995), Biarritz, Terres et Hommes du Sud, 1996, p. 67-85.

15. S. Trocmé Sweany, Estienne Pasquier (1529-1615) et nationalisme littéraire, Paris-Genève, Champion-Slatkine, 1985.

16. É. Pasquier, Euvres, cit., t. II, col 4.

17. Id., Le Monophile, Paris, E. Groulleau, 1554.

18. L'intitulé Epistres va changer, par volonté de Pasquier, dès 1610, en Lettres amoureuses. Nous pouvons reconnaître dans cette modification une astuce éditoriale et un clin d'œil à une typologie de lettres de dérivation italienne (les Lettere amorose), genre à la mode et de plus en plus répandu dans les recueils français.

19. É. Pasquier, Lettres amoureuses, I, dans Euvres, cit., t. II, col. 805.

20. Ibid., col. 805-806.

21. L'ouverture à la fiction est l'aspect le plus intéressant des Lettres amoureuses; voir, entre autres, B. Bray, "Les “lettres amoureuses” d'Étienne Pasquier, premier roman épistolaire français ? ", dans Cahiers de l'A.I.E.F., 29, 1977, p. 133-145 et M. Campanini, « Quand l'auteur se fait personnage. Le moi et le récit dans les "Lettres amoureuses" d'Étienne Pasquier (1555) », dans É. Pasquier, Épistolaire, 35, 2009, p. 37-48.

22. Voir É. Pasquier, CEuvres, cit., t. II, liv. II, Lettre 2, col. 30 : « Ceste maniere de faire n'a pas pleu au bon homme Erasme, qui veut que sans fiction une epistre ait été envoyée. Et quant à moy, son jugement ne me plaist, parce qu'estant cecy pratiqué de la façon que je dis, il apportera profit \& plaisir ensemble »

23. Lettre à M. Bigot, ibidem.

24. Voir Ibid., liv. VI, Lettre 3, col. 157 : «Je ne sçay si j'ay en cecy failli, mais s'il y a de ma faulte, elle est double : l'une d'avoir failly, l'autre de ne m'en pouvoir repentir »

25. Ibid., VI, 4, col. 157.

26. La seconde édition des Epistres paraît à l'intérieur de G. Parabosco, Lettres amoureuses de mess. Girolam. Parabosque, traduites d'italien en françois par Philip-Hubert de Villiers, Paris, G. Corrozet, s.d. Elles sont reprises en 1610 dans É. Pasquier, La Jeunesse d'Estienne Pasquier et sa suite, Paris, J. PetitPas, 1610 sous le titre - désormais définitif - de Lettres amoureuses et, par la suite, au t. III de É. Pasquier, CEuvres meslees d'Estienne Pasquier, Paris, J. Petit-Pas, 1619. Les Lettres amoureuses paraissent enfin dans CEuvres, cit., t. II.

27. V. C. Magnien-Simonin, "Étienne Pasquier (1529-1615) ou la dissidence discrète ", dans Les Dossiers du Grihl, 2013, consulté le 20/11/2017, URL : <http://dossiersgrihl.revues.org/5748>.

28. É. Pasquier, Euvres, cit., t. II, liv. VI, Lettres 3 et 4, col. 157.

29. «À Monsieur Airault Lieutenant Criminel au siège Presidial d'Angers » (Tours, 1591), dans Id., La Jeunesse d'Estienne Pasquier et sa suite, Paris, Jean Petit-Pas, 1610, p. 473-475. Voir aussi la lettre à M. Pitou (Ibid., p. 491), qui est une sorte de postface de la pastorale.

30. Au Lecteur, dans Ibid., f. A2 ${ }^{\circ}$.

31. Ibid., f. $\mathrm{A} 2 \mathrm{v}^{\circ}-\mathrm{A} 3 \mathrm{r}^{\circ}$.

32. Id., Cuvres, t. II, liv. XXI, Lettre 6, col. 648 : « Je ne voulois advoüer le recueil comme venant de ma boutique ; ainsi le verrez-vous en l'epistre liminaire par moy faite sous le nom d'un André du Chesne».

33. Ibidem : «[...] je ne me mets sur les rangs pour plaire seulement à ceux de ce temps, ains la postérité, si j'y puis atteindre, qui ne jugera s'il y a eu de la bien ou de la malseance en l'Autheur, la faisant imprimer, ains si l'ouvrage est de mérite ou non ».

34. «Les jeus poetiques d'Estienne Pasquier », dans Id., La jeunesse, cit., p. 332.

35. Ibidem. 
36. Dans la lettre à Saincte Marthe du $1^{\mathrm{er}}$ janvier 1613, Pasquier admet que ce recueil lui plaît, «comme l'enfant fait au père» et, plus loin, sur un ton plaisant, il s'autorise, en tant que vieillard, à être babillard et à se louer. Voir Id., CEuvres, t. II, liv. XXI, Lettre 6, col. 648.

37. Id., Euvres, t. II, liv. I, Lettre 1, col. 3.

38. Ibid., col. 649.

39. A. Furetière, "Discours sur l'art épistolaire ", dans Essais de Lettres familières sur toute sorte de Sujets, Paris, Le Febvre, 1690, p. 216.

40. Rigoley de Juvigny, Bibliothèques françoises de La Croix du Maine et Du Verdier, t. 3, Paris, Saillant et Nyon, 1772, p. 519.

41. Abbé Gouget, Bibliothèque françoise ou Histoire de la Littérature française, t. XIV, Paris, Guérin, 1752, p. 253-265.

42. Sainte-Beuve, Causeries du lundi, t. III, Paris, Garnier frères, p. 249-269, p. 254.

43. É. Pasquier, La jeunesse, cit., f. A2vº

\section{RÉSUMÉS}

Le vaste corpus de lettres d'Étienne Pasquier dont on analyse ici une sélection forcément très réduite mais traversant la production de l'auteur de 1555 (Recueil des rymes et proses) à 1610 (La jeunesse) offre une matière privilégiée à une enquête sur les dispositifs et les enjeux de la parole auctoriale au moment où celle-ci s'applique à porter un jugement sur l'œuvre et sur les spécificités de l'énonciation épistolaire, à dessiner l'ethos d'auteur et à tracer les lignes d'une stratégie éditoriale. À travers l'analyse de quelques péritextes épistolaires et de quelques lettres ayant fonction d'autocommentaire, on assiste à l'émergence du discours d'auteur et de l'image d'une œuvre et d'une écriture dominées par la variété et la plasticité et imbues de subjectivité, ce qui favorise parfois l'inflexion vers le champ de la fiction, notamment dans les lettres amoureuses. Au fil de la réflexion métadiscursive de Pasquier on suit la construction et la promotion d'une conscience littéraire qui se veut l'interprète d'un renouveau de la forme-lettre et de la promotion d'une prose épistolaire française vue comme modèle pour une littérature nationale. Il s'agit finalement d'une autocritique d'auteur destinée à laisser son empreinte sur la réception de l'œuvre.

\section{INDEX}

Mots-clés : Pasquier (Étienne), épistolarité, Lettre familière, Renaissance, autocritique 